Revista Digital Universitaria

Vol. 23, Núm. 1, enero-febrero 2022

\title{
Cultivar la diversidad e inclusión en la educación
}

Verónica Cervantes López

\section{Resumen}

A través de los años, la educación en México ha tenido constantes cambios debido a las diferentes reformas educativas, con la visión de elevar la calidad educativa, tomando en cuenta la diversidad e inclusión de los alumnos en todos los ámbitos del contexto escolar donde se desenvuelven. Resulta inquietante lo que será la educación dentro de pocos años con un sistema educativo justo e incluyente, ya que en la actualidad se llevan a cabo diversas estrategias de oportunidad para que los alumnos sean tomados en cuenta y ver la diversidad de cada uno como herramienta que favorezca el proceso de enseñanza aprendizaje y engrandecer las culturas y diferencias.

Palabras clave: Diversidad, identidad, inclusión, educación intercultural.

\section{Cultivate Diversity AND INCLUSION IN EDUCATION}

\begin{abstract}
Throughout the years, education in Mexico has had constant changes due to different educational reforms, with the vision of raising educational quality, considering the diversity and inclusion of students in all areas of the school context where they operate. It is disturbing what education will be in a few years with a fair and inclusive educational system, since at present various strategies of opportunity are carried out so that students are taken into account and see the diversity of each one as a tool that help the teaching-learning process and enhance cultures and differences.
\end{abstract}

Keywords: Diversity, identity, inclusion, intercultural education. 
Licenciada en Educación Secundaria con especialidad en Español, en la Escuela Normal Superior "José E. Medrano R." extensión Nuevo Casas Grandes; cuenta con una maestría en Estrategia Educativa cursada en la Universidad Regional del Norte de la Ciudad de Chihuahua, Chihuahua, en la cual, cursa el doctorado en Educación. Actualemente es docente en la Escuela Secundaria Técnica No. 24 ubicada en el municipio de Janos, Chihuahua; sus intereses de investigación están relacionados con la vulnerabilidad de los adolescentes, los espacios de convivencia, así como la diversidad de alumnos presente en las escuelas.

\section{Introducción}

U no de los factores que enfrenta el ámbito educativo es la diversidad de alumnado que atiende, por lo que es conveniente aprovechar la riqueza que se tiene ante esas diferencias para convertirlas en fortalezas y para que cada centro educativo lo aborde de una manera que repercuta favorablemente en el proceso educativo, así como también en los integrantes de las familias y por consecuencia en la sociedad en general.

De esta manera, "La inclusión en el ámbito de la educación no sólo postula el derecho de las personas a ser diferentes como algo legítimo, sino que valora explícitamente la existencia de esa diversidad y promueve el trato equitativo". (Secretaría de Educación Pública [SEP], 2017), promoviendo con ello que los estudiantes conserven tanto sus raíces como su esencia.

Un sistema educativo justo e incluyente constituye una de las palancas más eficaces para la transformación de la sociedad. Como señala Solari, para que la educación de calidad pueda tener el efecto de producir menor desigualdad, ella misma debe ser lo más igualmente distribuida posible. Cuando eso no ocurre, la desigualdad educativa limita la posibilidad de que niñas, niños y jóvenes en desventaja puedan salir de esa situación. (SEP, 2017).

Sin duda, los estudiantes al sentirse valorados sin importar la diversidad que se presente, se desenvolverán de una forma positiva, con una mejor autoestima y con la visión de apoyar a sus pares. 


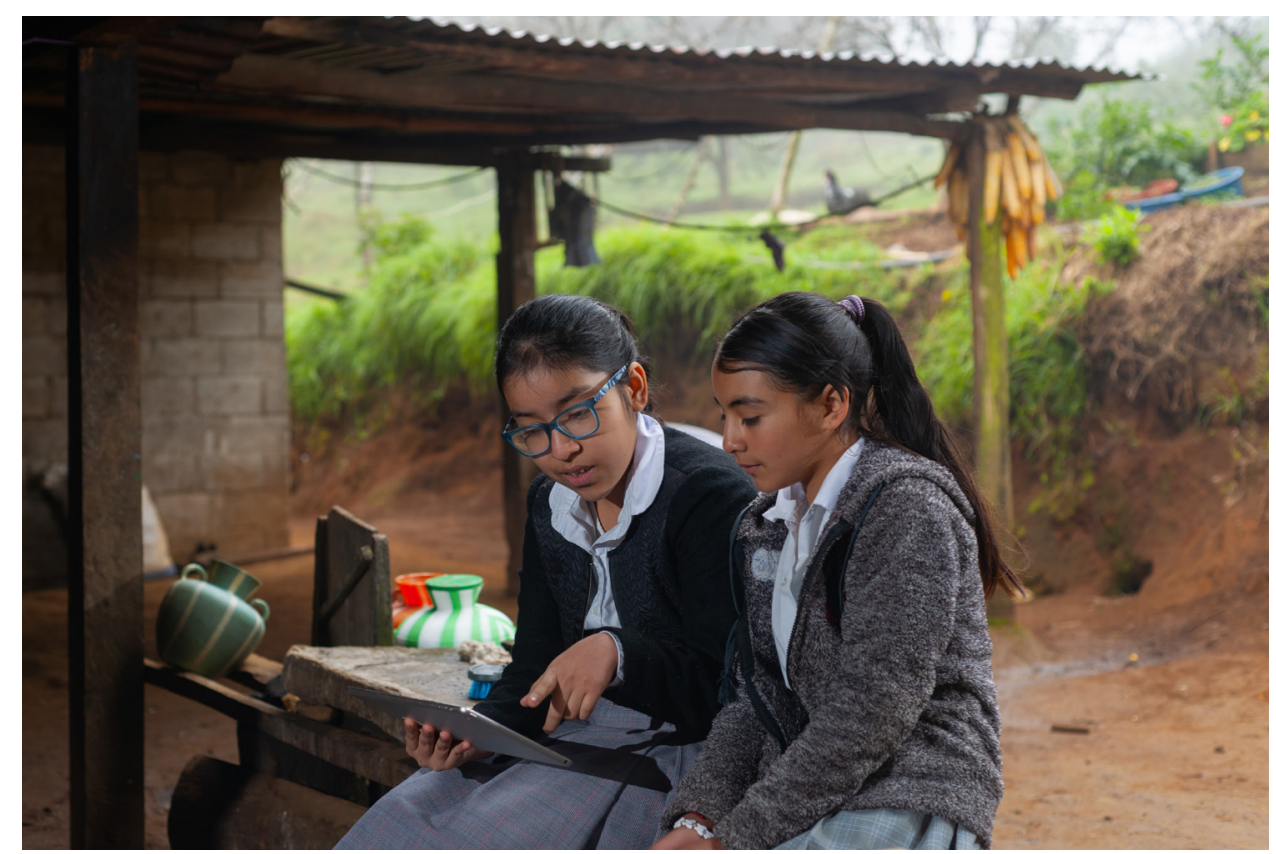

\section{Desarrollo}

El ser humano por naturaleza es un ser social, nace, crece y se reproduce dentro de varios ambientes, formándose para ser parte de una sociedad y dadas las características de la misma, lo va a formar como individuo. Por esto, cuando entra al ámbito educativo ya tiene una personalidad propia que va definiéndose con el transcurrir del tiempo hasta llegar a la meta, aunque esta sea corta en la mayoría de los casos. No todos los que ingresan al nivel medio superior logran terminar o continuar con sus estudios, una de las causas es la discriminación que viven por la diversidad que presenta cada uno y que resulta difícil adaptarse a un contexto de inequidad.

De acuerdo con los indicadores de media superior, la tasa de eficiencia terminal es del 66.7\%, el 71.3\% mujeres y 62.2\% hombres; teniendo así un porcentaje de abandono escolar total de 10.3\%, 8.3 mujeres y $12.5 \%$ hombres. (SEP, 2021). En estos datos se incluyen las modalidades escolarizada y no escolarizada.

Indudablemente, como lo menciona Morales (2015) "lo que diferencia a cada ser humano es importante no solo de una forma individual, sino en interacción con las particularidades de las otras personas, punto clave dentro de los planteamientos de la interculturalidad en el abordaje de la diversidad" (p.4). Es necesario ver la educación como una posibilidad de cambio y de resolución de problemáticas que se presentan. 
El docente ocupa un espacio muy importante en la vida diaria de cada alumno, porque contribuye con otra parte de la educación que es la de auxiliar con herramientas que ayuden a formarlo como individuo, para que se incorpore a una vida democrática y aprenda a salir adelante por sí mismo. Como tal, el educador deberá tomar actitudes positivas y empáticas que conlleven a una relación fructífera, y el educando cada día muestre más interés por el trabajo áulico. Deberá ser guía, apoyo, empático, tenaz, audaz para dar seguimiento a esta ardua tarea que es la de formar personas con principios y valores bien cimentados que se lleven a la práctica diaria en la vida cotidiana.

Para llegar a plenitud como seres humanos se necesita de otros. Aprender a vivir juntos es indispensable para realizar cualquier actividad.

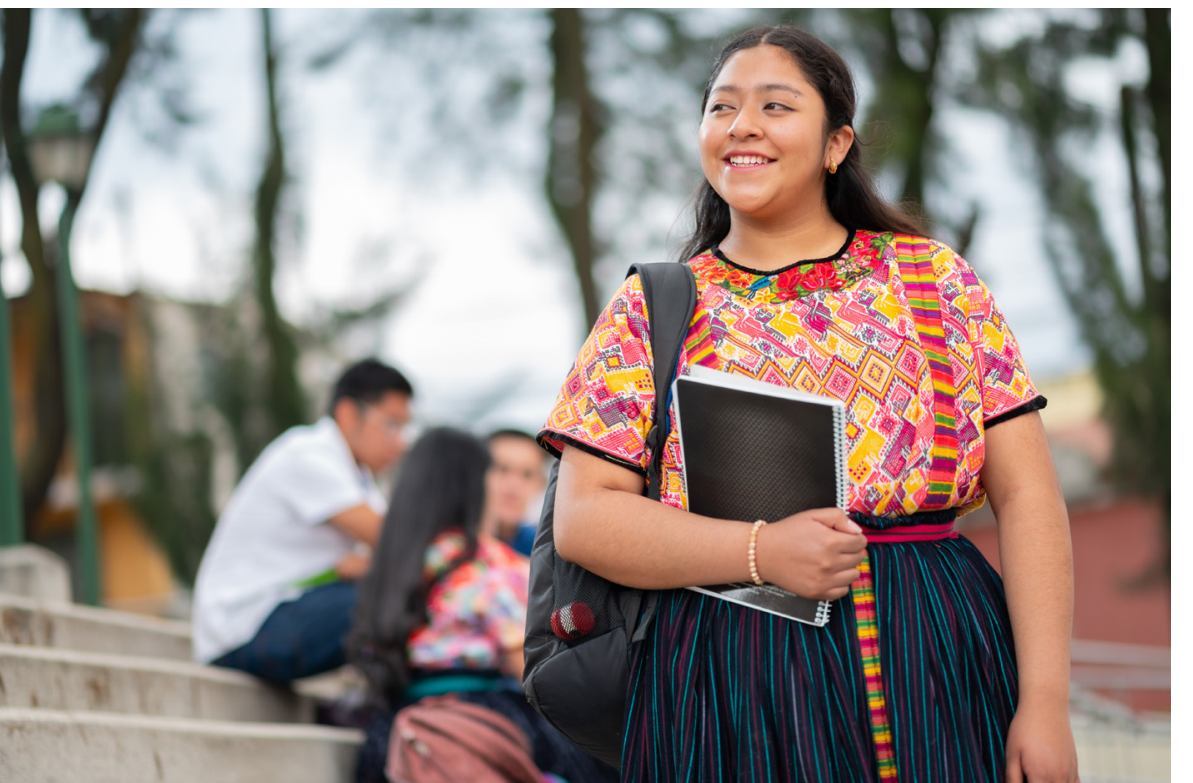
la educación y que se debe brindar de la mejor manera para que su desarrollo sea eficaz y de calidad.

Es por lo anterior que los docentes tienen un gran compromiso de ayudar a que los educandos se desenvuelvan de forma inclusiva, con habilidades y tomen en cuenta que es necesario estar en constante actualización para poder cumplir con los requerimientos que la labor demanda.

"Es importante que, desde los procesos de formación tanto inicial como permanente, se promueva entre el profesorado la adquisición de las competencias necesarias para dar respuesta a los retos que supone la Educación Intercultural". (Figueredo y Ortíz, 2014).

Entendiendo por dichas competencias interculturales, aquellas que permiten a las personas convivir en una sociedad diversa, así como comunicarse y relacionarse con los demás, también valorar las diferencias y expresar sus propias 
ideas; es muy importante practicar la empatía, la tolerancia y sobre todo el respetar los valores, creencias y la historia de cada alumno con el que socializan.

En definitiva, "es necesario cultivar actitudes interculturales, es decir, los docentes deben favorecer el desarrollo de competencias comportamentales en sus estudiantes a través de la formación de valores, tanto dentro como fuera del aula, hacia sus compañeros y personas adultas". (Alarcón y Márquez, 2019).

Harrisy Chapman; Muijs et al. (como se citó en Murillo et al., 2010), mencionan:

La educación inclusiva entendida como una escuela para la justicia social se rige por valores y principios tales como:

1. Integridad y honestidad.

2. Justicia y equidad.

3. La promoción del respeto por todos los individuos.

4. El reconocimiento de que todos los estudiantes tienen derecho a una educación basada en la igualdad de oportunidades.

5. Preocupación por el bienestar y el desarrollo integral de los profesores y los estudiantes.

6. Todos los estudiantes pueden aprender.

7. La escuela debe responder a las necesidades particulares de cada estudiante.

8. El punto de vista de los estudiantes es importante y tomado en cuenta.

9. La diferencia es vista como una oportunidad para aprender y como una fuente de enriquecimiento.

Cabe resaltar, que el trabajo en colectivo y la unificación de criterios no solo de los docentes sino de todos los que laboran en las instituciones educativas son imprescindibles para el desarrollo de una educación en la diversidad, se busca siempre que los resultados sean favorables y que de ello resulten experiencias exitosas que se puedan mejorar aún más con la práctica.

Con respecto al tema, la Constitución Política de los Estados Unidos Mexicanos (1917), hace referencia a la educación intercultural como un derecho de todo individuo.

Garantizar e incrementar los niveles de escolaridad, favoreciendo la educación bilingüe e intercultural, la alfabetización, la conclusión de la educación básica, la capacitación productiva y la educación media superior y superior. Establecer un sistema de becas para los estudiantes indígenas en todos los niveles. Definir y desarrollar programas educativos de contenido regional que reconozcan la herencia cultural de sus pueblos, de acuerdo con las leyes de la materia y en consulta con las comunidades indígenas. Impulsar el respeto y conocimiento de las diversas culturas existentes en la nación. (Art. $2^{\circ}$ B. II). 
En la todos de los planteles educativos se encuentran casos diversos, donde es necesario implementar estrategias para que se trabaje de una manera inclusiva el trato hacia los educandos y a la vez que ellos desarrollen habilidades donde se vea favorecido el proceso de enseñanza aprendizaje, así como las competencias que le servirán en la resolución de problemas que se presenten a lo largo de su vida, tal como se menciona en uno de los principios rectores que guían la Estrategia Nacional de Educación Inclusiva (ENEI) en materia de educación inclusiva y que hace referencia a:

Diversidad: se traduce en una multiplicidad de formas de pensamiento, sentimiento y cultura. Es inherente a cualquier comunidad humana, sea pequeña o grande. La diversidad es expresión de libertad y se encarna jurídicamente en el derecho a la diferencia. Las sociedades abiertas y democráticas la protegen y la cultivan. En la educación básica se pretende que la diversidad sea una ventaja en dos sentidos: por un lado, permite aprender a convivir y, por otro, promueve el desarrollo cognitivo de los educandos. (SEP, 2019, p. 76)

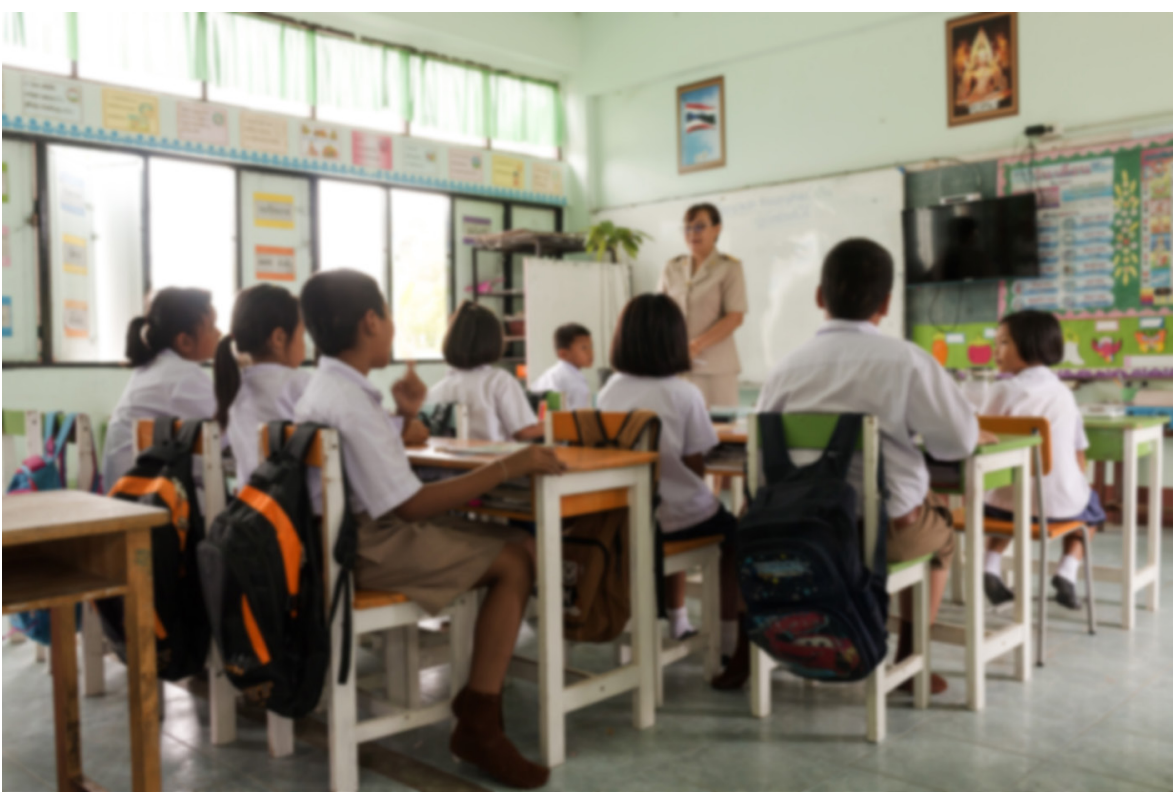

En relación a la idea anterior, es indispensable que se cuente en los planteles educativos con un liderazgo efectivo, ya que de ello depende en gran parte el éxito en el desarrollo de cualquier estrategia que se pretenda implementar, el seguimiento correspondiente a casos que se presenten, así como también para la gestión tanto de recursos y apoyos en beneficio de toda comunidad escolar.

Implementar estrategias de equidad e inclusión en el sistema educativo implica responder y enfrentar, desde los procesos formativos actuales, a las desigualdades que se gestaron en el pasado, incluso desde el propio sistema educativo, y que han impedido y limitado el crecimiento integral y la movilidad social de millones de personas en México. Indica un proceso de transformación en el que las escuelas se desarrollan y adaptan a la diversidad de los alumnos. La diversidad y las diferencias nos enriquecen y nos hacen partícipes de una sociedad que aspira cada día a ser más justa e incluyente. La heterogeneidad debe reconocerse como cualidad cotidiana más que excepcional en el sistema educativo, puesto que gracias a ellas podremos desarrollarnos como personas iguales en capacidades sin importar nuestras condiciones de origen. (SEP, 2017). 


\section{Conclusión}

Para que en México se desarrolle favorablemente la inclusión escolar y se acepte la diversidad en la educación, es imprescindible tener en cuenta la importancia del compromiso docente, así como de un liderazgo efectivo, para poder llevar a cabo la concientización sobre el gran trabajo que se puede realizar en conjunto con un fin común, que es el de mejorar la calidad educativa para que los alumnos sin importar su contexto, adquieran y desarrollen habilidades para un aprendizaje que le sirva para toda la vida.

Por lo antes expuesto, se puede llegar a las siguientes conclusiones del corto análisis sobre la diversidad e inclusión en la educación.

- Todo cambio, reforma o transformación es bueno, pero sin un seguimiento es muy difícil y nada satisfactorio; por lo que los docentes tienen la responsabilidad de ser flexibles y adoptar una actitud positiva ante los cambios que se presentan, con la finalidad de que los alumnos desarrollen habilidades dentro de un contexto donde se reconozca su diversidad.

- Aceptar los beneficios que puede tener la diversidad en todos sus aspectos dentro del contexto escolar.

- Estar todos los docentes en el mismo canal y buscar las estrategias adecuadas para en realidad llevar a cabo la inclusión con equidad y eficacia.

- Como docentes, estar en constante actualización, para un mejor desarrollo de los educandos.

- Buscar como trabajadores de la educación, la manera de que el alumno se vaya apropiando de conocimientos basados en su interés y necesidad; así como también, implementar la equidad educativa.

- Que nuestras autoridades acepten realmente el cambio que se requiere, para el bienestar de todos los inmiscuidos en el ámbito educativo, pero principalmente el de los educandos, ya que ellos son el futuro de nuestro México.

- Contar en la institución educativa con un liderazgo efectivo, que guíe, gestione y sobre todo apoye en todo momento las acciones que deban realizarse para llevar a cabo la educación en la diversidad.

Hablar de equidad e inclusión es referirnos a un método que invita a la reflexión permanente sobre cómo transformar y mejorar los sistemas educativos, con el propósito de que respondan y atiendan la riqueza personal, social y cultural de los estudiantes. (SEP, 2017). 


\section{Referencias}

* Alarcón, J. y Márquez, J. (2019). Competencias docentes interculturales. Multiculturalidad y consecuencias para la inmigración. Scielo, 45(2). https://scielo. conicyt.cl/pdf/estped/v45n2/0718-0705-estped-45-02-7.pdf

* Constitución Política de los Estados Unidos Mexicanos (5 de febrero de 1917). http://www.diputados.gob.mx/LeyesBiblio/pdf/1_280521.pdf

* Figueredo, V. y Ortiz, L. (2014). Desarrollo de competencias interculturales en la formación del profesorado en Andalucía. Journal for Educators, Teachers and Trainers, 5(2), 160 - 179. http://www.ugr.es/ jett/pdf/Vol5(2)_012_jett_Figueredo_ Ortiz.pdf

* González, M. (2008). Diversidad e inclusión educativa: Algunas reflexiones sobre el liderazgo en el Centro Escolar. REICE, 6(2). https://revistas.uam.es/index.php/ reice/article/view/5443

* Morales, C. (2015). Diversidad juvenil en el contexto educativo: Reflexiones para un abordaje intercultural. Revista Electrónica "Actualidades Investigativas en Educación", 15(1). https://www.redalyc.org/pdf/447/44733027023.pdf

* Murillo, F., Krichesky, A., Castro, A. y Hernández, R. (2010). Liderazgo para la inclusión escolar y la justicia social. Aportaciones de la investigación. Revista Latinoamericana de Patología, 4(1), 169-187. https://cutt.ly/7lomjc3

* Secretaría de Educación Pública [SEP]. (2017). Modelo Educativo. Equidad e inclusión. https://www.gob.mx/cms/uploads/attachment/file/283701/E_Equidade-inclusion_0717.pdf

* Secretaría de Educación Pública [SEP]. (2019). Estrategia Nacional de Educación Inclusiva. https://www.animalpolitico.com/wp-content/uploads/2019/11/ENEl.pdf

* Secretaría de Educación Pública [SEP]. (2021). Principales Cifras del Sistema Educativo Nacional 2020-21. https://www. planeacion.sep.gob.mx/

\section{Obras consultadas}

* Centro de Escritura Javeriano. (2020). Normas APA, séptima edición. Pontificia Universidad Javeriana, seccional Cali. https://cutt.ly/ulpxKpr

\section{Cómo citar este artículo}

* Cervantes López, Verónica. (2022, enero-febrero). Cultivar la diversidad e inclusión en la educación. Revista Digital Universitaria (RDU), 23(1). http://doi.org/10.22201/ cuaieed.16076079e.2022.23.1.5 